\title{
CORRIGENDUM
}

\section{Avoiding early closing: \\ 'Livšic theorems for non-commutative groups including diffeomorphism groups and results on the existence of conformal structures for Anosov systems' - CORRIGENDUM}

\author{
RAFAEL DE LA LLAVE and ALISTAIR WINDSOR
}

doi:10.1017/S014338570900039X, Published by Cambridge University Press, 17 July 2009.

\section{Introduction}

This paper serves as a corrigendum to our paper [dILW09]. In particular, in the proof of Theorem 6.3 we claimed the following.

CLAIM. Let $f$ be a topologically transitive Anosov diffeomorphism of a compact manifold $M$. For all $\epsilon>0$ there exists $L \geq 1$ such that for every $n \in \mathbb{N}$ and $x \in M$ there exists $a$ periodic point $p \in M$ satisfying:

(1) for all $0 \leq i \leq n$,

$$
d_{M}\left(f^{i} x, f^{i} p\right)<\epsilon
$$

and

(2) $\quad p$ has minimal period $n+\ell$ with $0 \leq \ell \leq L$.

Unfortunately, as B. Kalinin and V. Sadovskaya discovered, the proof sketched contained gaps. Using specification as was suggested in our paper leads to a weaker result than we claimed. In this paper we prove a uniform version of closing.

THEOREM. Let $f$ be a topologically transitive $C^{1}$ Anosov diffeomorphism of a compact connected manifold $M$. Given $\epsilon>0$ there exists $D \geq 1$ and $N>0$ such that for all $x \in M$ and $n \in \mathbb{N}$ there exists a periodic point $p \in M$ with minimal period $m \in \mathbb{N}$ and $d \in \mathbb{N}$ such that:

(1) for all $0 \leq i \leq n-1$

$$
d_{M}\left(f^{i} x, f^{i} p\right)<\epsilon
$$

and

(2) $n \leq d \cdot m \leq n+N$ and $1 \leq d \leq D$.

This result is strong enough to complete the proof of Theorem 6.3. 


\section{Results}

To prove our result for Anosov diffeomorphisms, we will first prove a similar statement for subshifts of finite type. Since every Anosov diffeomorphism is a factor of a subshift, this will allow us to establish the desired result.

Recall that a subshift of finite type can be described by a transition matrix $A$. Symbol $j$ may follow symbol $i$ in a word in $\Sigma_{A}$ if $A_{i, j}=1$. A finite sequence $\left(a_{1}, \ldots, a_{n}\right)$ is said to be admissible if $A_{a_{i}, a_{i+1}}=1$ for $0 \leq i \leq n-1$. We say that a finite sequence $\left(a_{1}, \ldots, a_{n}\right)$ is periodic if it is admissible and $A_{a_{n}, a_{1}}=1$ so that the sequence can be extended periodically to a point $a \in \Sigma_{A}$ of period $n$.

The following result is similar to one of Fine and Wilf in [FW65].

LEMMA 1. Let $\left(\Sigma_{A}, \sigma\right)$ be a subshift of finite type. Let $\left(a_{1}, \ldots, a_{n}\right)$ be a periodic sequence of period $m_{1}$. Let $\left(a_{1}, \ldots, a_{n}, \ldots, a_{n+L}\right)$ be an extension of $\left(a_{1}, \ldots, a_{n}\right)$ that is periodic with period $m_{2}$.

If $m_{1}+m_{2} \leq n$, then $\left(a_{1}, \ldots, a_{n}\right)$ and $\left(a_{1}, \ldots, a_{n+L}\right)$ are both periodic of period $\operatorname{gcd}\left(m_{1}, m_{2}\right)$.

Proof. Write $d:=\operatorname{gcd}\left(m_{1}, m_{2}\right)=k_{1} m_{1}+k_{2} m_{2}$ with $k_{1}, k_{2} \in \mathbb{Z}$. Consider the following variation on the proof of Bézout's theorem that uses only numbers in the range $1, \ldots, n$. If $k_{1}>0$, then define $k_{+}=k_{1}, m_{+}=m_{1}, k_{-}=-k_{2}$ and $m_{-}=m_{2}$. If $k_{2}>0$, then define $k_{+}=k_{2}, m_{+}=m_{2}, k_{-}=-k_{1}$ and $m_{-}=m_{1}$.

Let $1 \leq i \leq n-d$ be arbitrary and initialize $k=i$.

(1) Add $m_{+}$to $k$ successively until either of the following holds:

(a) adding a further $m_{+}$would give $k$ above $n$; or

(b) all $k_{+}$of the $m_{+}$have been used.

(2) Subtract $m_{-}$from the new $k$ successively until either of the following holds:

(a) subtracting a further $m_{-}$would give $k$ below 1 ; or

(b) all $k_{-}$of the $m_{-}$have been used.

(3) If $k \neq i+d$, then return to step (1).

Notice that if $k+m_{+} \geq n+1$ and $k-m_{-} \leq 0$, then $m_{1}+m_{2} \geq n+1$, which is a contradiction. Thus the above procedure cannot terminate at an intermediate stage, and the algorithm must proceed to give $k=i+d$.

Since each of these steps involves one of the two periods and all of the numbers are among $1, \ldots, n$, this shows that $a_{i}=a_{i+d}$ for $1 \leq i \leq n-d$, i.e. the original sequence $\left(x_{1}, \ldots, x_{n}\right)$ is $d$-periodic. Since $d$ divides $m_{2}$ and $m_{2}<n$, we can conclude that the extended sequence $\left(a_{1}, \ldots, a_{n+L}\right)$ is also $d$-periodic.

Remark. The hypothesis that $m_{1}+m_{2} \leq n$ is necessary. If we take the sequence

$$
(0,1,0,1,0,0,1,0,1,0)
$$

of length 10 with period 5 and extend it by $(1,0,0,1)$, we obtain the sequence

$$
(0,1,0,1,0,0,1,0,1,0,1,0,0,1)
$$

of length 14 that has period 7. Obviously, the original sequence is not constant even though 5 and 7 are relatively prime. 
THEOREM 1. Let $\left(\Sigma_{A}, \sigma\right)$ be a mixing subshift of finite type. Let $L$ be such that $A^{L}>2$. Let $n \geq L$. Let $\left(a_{1}, \ldots, a_{n}\right)$ be a periodic sequence. Then one of the following holds:

(1) $\left(a_{1}, \ldots, a_{n}\right)$ has minimal period $n$ or $n / 2$; or

(2) there exists an extension $\left(a_{1}, \ldots, a_{n+L}\right)$ of $\left(a_{1}, \ldots, a_{n}\right)$ such that $\left(a_{1}, \ldots, a_{n+L}\right)$ is periodic with minimal period $n+L$ or $(n+L) / 2$.

Proof. If $\left(a_{1}, \ldots, a_{n}\right)$ has minimal period $m_{1}$ being either $n$ or $n / 2$, then we are done; therefore we may suppose that $m_{1} \leq n / 3$. Let $\left(a_{1}, \ldots, a_{n+L}\right)$ be an arbitrary periodic extension of $\left(a_{1}, \ldots, a_{n}\right)$. If $\left(a_{1}, \ldots, a_{n+L}\right)$ has minimal period $m_{2}$ being either $n+L$ or $(n+L) / 2$, then we are done; therefore we may suppose that $m_{2} \leq(n+L) / 3$.

In this case, since $L \leq n$ we must have $m_{1}+m_{2} \leq n$. Hence, by Lemma 1 , we can show that the extended sequence $\left(a_{1}, \ldots, a_{n+L}\right)$ has period $m_{1}$. There is a unique extension of $\left(a_{1}, \ldots, a_{n}\right)$ that makes $\left(a_{1}, \ldots, a_{n+L}\right)$ have period $m_{1}$, but there are at least two ways of completing $\left(a_{1}, \ldots, a_{n+L}\right)$. Using this other completion, we get that $\left(a_{1}, \ldots, a_{n+L}\right)$ does not have period $m_{1}$. If we denote its minimal period by $m_{2}$, we see that we must have $m_{1}+m_{2}>n$. This means that $m_{2}$ must be at least $(n+L) / 2$.

Now we can state our main theorem.

THEOREM 2. Let $f$ be a topologically transitive $C^{1}$ Anosov diffeomorphism of a compact connected manifold $M$. Given $\epsilon>0$, there exist $D \geq 1$ and $N>0$ such that for all $x \in M$ and $n \in \mathbb{N}$ there exist a periodic point $p \in M$ with minimal period $m \in \mathbb{N}$ and $d \in \mathbb{N}$ such that:

(1) for all $0 \leq i \leq n-1$,

$$
d_{M}\left(f^{i} x, f^{i} p\right)<\epsilon
$$

and

(2) $n \leq d \cdot m \leq n+N$ and $1 \leq d \leq D$.

Proof. Let $\epsilon>0$ be arbitrary. There exists a Markov partition $\mathfrak{M}$ of $M$ by 'rectangles' of diameter less than $\epsilon$ (see [Bow70b]). Let $\left(\Sigma_{A}, \sigma\right)$ be the associated subshift of finite type with transition matrix $A$ and alphabet $\mathscr{A}$. Every transitive Anosov diffeomorphism of a connected manifold is topologically mixing, so there exists $L \in \mathbb{N}$ such that $A^{L}$ is a positive matrix. Immediately, we have $A^{2 L} \geq 2$. By [Bow70a, Proposition 10], there exists $k \in \mathbb{N}$ such that the canonical projection $\pi: \Sigma_{A} \rightarrow M$ satisfies $\# \pi^{-1}(x) \leq k$ for all $x \in M$.

Consider one of the possible lifts of the point $x \in M,\left(\ldots, x_{0}, x_{1}, \ldots, x_{n-1}, \ldots\right)$. Consider the finite sequence $\left(x_{0}, \ldots, x_{n-1}\right)$. We can extend this by $2 L$ states to get a new finite sequence $\left(y_{0}, \ldots, y_{n-1+2 L}\right)$ that is periodic; we choose to extend by $2 L$ rather than simply $L$ so that $2 L<n+2 L$. Now we can apply our symbolic extension lemma to obtain either a point $q$ of period $n+2 L$ with minimal period at least $(n+2 L) / 2$ or a point $q$ of period $n+4 L$ with minimal period $(n+4 L) / 2$. Let $m$ be the minimal period of the point $q$. The orbit of the periodic point $q$ consists of $m$ distinct points. Projecting the 
orbit under $\pi$ gives at least $m / k$ distinct points. Hence the minimal period of the projected point $p=\pi(q)$ is at least $m / k$.

Taking $N=4 L$ and $D=2 k$, we see that we obtain a periodic point $p$ of period $n \leq n^{\prime} \leq n+N$ with minimal period at least $n^{\prime} / D$.

Since we extended the original $\left(x_{0}, \ldots, x_{n-1}\right)$, we have that $p$ and $x$ belong to the same rectangle for the first $n$ iterations of $f$. This means that

$$
d_{M}\left(f^{i} x, f^{i} p\right)<\epsilon
$$

for $0 \leq i \leq n-1$.

\section{Completing the proof of Theorem 6.3}

Theorem 6.3 states that if the distortion of $f$ along every periodic orbit is bounded, then the distortion of any iterate of $f$ is uniformly bounded. The idea was that any orbit segment is close to a segment of a periodic orbit whose period is not very different from the length of the orbit segment. This led to the inequalities (54) in [dILW09]:

$$
\begin{aligned}
K_{g, E^{s}}\left(f^{n}, p\right) & \leq K_{g, E^{s}}\left(f^{n+\ell}, p\right) K_{g, E^{s}}\left(f^{-\ell}, p\right) \\
& \leq C_{\text {per }} K_{g, E^{s}}\left(f^{-\ell}\right) .
\end{aligned}
$$

Here we used $K_{g, E^{s}}\left(f^{n+\ell}, p\right) \leq C_{\text {per }}$, since we were supposing that $n+\ell$ was the minimal period of the periodic point $p$. We are unable to show that this is the case; however, using our previous lemma, we can find a periodic point $p$ with minimal period $m$ such that for some $d \in \mathbb{N}$ with $1 \leq d \leq D$ we have $m \cdot d=n+\ell$ for $0 \leq \ell \leq N$. Now we have

$$
\begin{aligned}
K_{g, E^{s}}\left(f^{n+\ell}, p\right) & =K_{g, E^{s}}\left(f^{m \cdot d}, p\right) \\
& =K_{g, E^{s}}\left(f^{m}, p\right)^{d} \\
& \leq K_{g, E^{s}}\left(f^{m}, p\right)^{D} \\
& \leq C_{\mathrm{per}}^{D} .
\end{aligned}
$$

This then leads immediately to the following replacement for (54):

$$
\begin{aligned}
K_{g, E^{s}}\left(f^{n}, p\right) & \leq K_{g, E^{s}}\left(f^{n+\ell}, p\right) K_{g, E^{s}}\left(f^{-\ell}, p\right) \\
& \leq C_{\text {per }}^{D} K_{g, E^{s}}\left(f^{-\ell}\right) .
\end{aligned}
$$

With estimate $\left(54^{\prime}\right)$, the remainder of the proof of [dILW09, Theorem 6.3] carries through as stated. The only change is in the value of the constant obtained.

Acknowledgement. R. de la Llave was supported by NSF grant DMS-0901389.

\section{REFERENCES}

[Bow70a] R. Bowen. Markov partitions and minimal sets for axiom A diffeomorphisms. Amer. J. Math. 92 (1970), 907-918.

[Bow70b] R. Bowen. Markov partitions for axiom A diffeomorphisms. Amer. J. Math. 92 (1970), 725-747.

[dILW09] R. de la Llave and A. Windsor. Livšic theorems for non-commutative groups including diffeomorphism groups and results on the existence of conformal structures for Anosov systems. Ergod. Th. \& Dynam. Sys. 30(4) (2009), 1055-1100. Published online by Cambridge University Press 17 July 2009, doi: 10.1017/S014338570900039X.

[FW65] N. J. Fine and H. S. Wilf. Uniqueness theorems for periodic functions. Proc. Amer. Math. Soc. 16 (1965), 109-114. 